\title{
The use of an axicon beam-shaping element in non-modulated pyramid wavefront sensors
}

\author{
Guillaume Allain ${ }^{1}$, Denis Brousseau ${ }^{1}$, Simon Thibault ${ }^{1}$, and Jean-Pierre Véran ${ }^{2}$ \\ ${ }^{1}$ Université Laval, Centre d'optique, photonique et laser, Québec, Canada \\ ${ }^{2} \mathrm{CNRC}$ Herzberg, Institute of Astrophysics, Victoria, Canada
}

\begin{abstract}
Coronagraphs designed for high contrast imaging need high performance adaptive optics correction in order to reach typical contrast ratios requirements of about $10^{-9}$. The use of non-modulated pyramid wavefront sensors allows adaptive optics systems tomeasure wavefront errors with a high sensitivity, allowing such high contrast ratios. The lack of modulation, however, makes them more sensitive to the pyramid defects. It is notably difficult to find a pyramid on which the apex is manufactured to a high enough quality to allow its use in a non-modulated system.

We propose an innovative way to counter this problem, in the situation where the telescope has a central obscuration, by adding known non-common path aberrations to the wavefront by means of a beam shaping optical component. This component (e.g. axicon) is placed at a position conjugate to the telescope pupil plane and the resulting image plane is incident onto a standard pyramid. This element shapes the point spread function (PSF) from a spatially compact point to a shape that has minimal light at the center. This insures that next to no light is incident on the pyramid apex where most of the defects are present. The images of the sub-pupils are then used to retrieve the wavefront aberration using a similar way to what is done with a typical pyramid wavefront sensor.
\end{abstract}

Keywords: Pyramid Wavefront Sensor, Static modulation, Beam shaping, Axicon, High contrast imaging

\section{INTRODUCTION}

The new generation of high contrast imaging instruments have very demanding specification on the stability of the wavefront that is incident on the coronagraphs ${ }^{1}$ in order to detect earth-like exoplanets. ${ }^{2}$ The adaptive optics systems integrated in such instruments need high performance wavefront sensing in order to correct the wavefront error to an acceptable level to achieve $10^{-9}$ contrast. The use of a low-order wavefront sensor (LOWFS) becomes necessary to increase stability error caused by incorect pointing, vibrations or atmospheric turbulence. The solutions proposed (Zernike wavefront sensor, Lyot-Based wavefront sensor, etc.. ) aren't suited smaller project with less available light or need for tunable sensitivity range. Also, in a small scale project where white light need to be used in order to correct to a high enough frequency to achieve the contrast requirements, it is necessary to have a system which can be made achromatic as much as possible, which is simply impossible with some of the solutions proposed.

The present article proposes a new type of LOWFS based on a fixed pyramid wavefront sensor (FPWFS) that uses beam-shaping as an answer to the problems of current systems by using a known non-common path aberration. We will first present the reasons behind the use of an axicon as a beam-shaping element in the FPWFS system, which is focused around concerns around the manufacturing defects in pyramid apexes and very limited reconstruction linearity range. The results of fourier-based computer simulation will be analysed to insure that the wavefront sensor is reconstructing aberrations as intended. As it will be possible to see, these objects based python simulations were developed for this exact task to include everything that could be of a concern on a real system. We will conclude by presenting the current state of the project and pathway to integrate the described LOWFS in a complete astronomy system.

Further author information: (Send correspondence to Guillaume Allain)

Guillaume Allain: E-mail: guillaume.allain.1@ulaval.ca 


\section{PRINCIPLES OF THE BEAM-SHAPING PYRAMID WAVEFRONT SENSOR}

\subsection{Pyramid wavefront sensor}

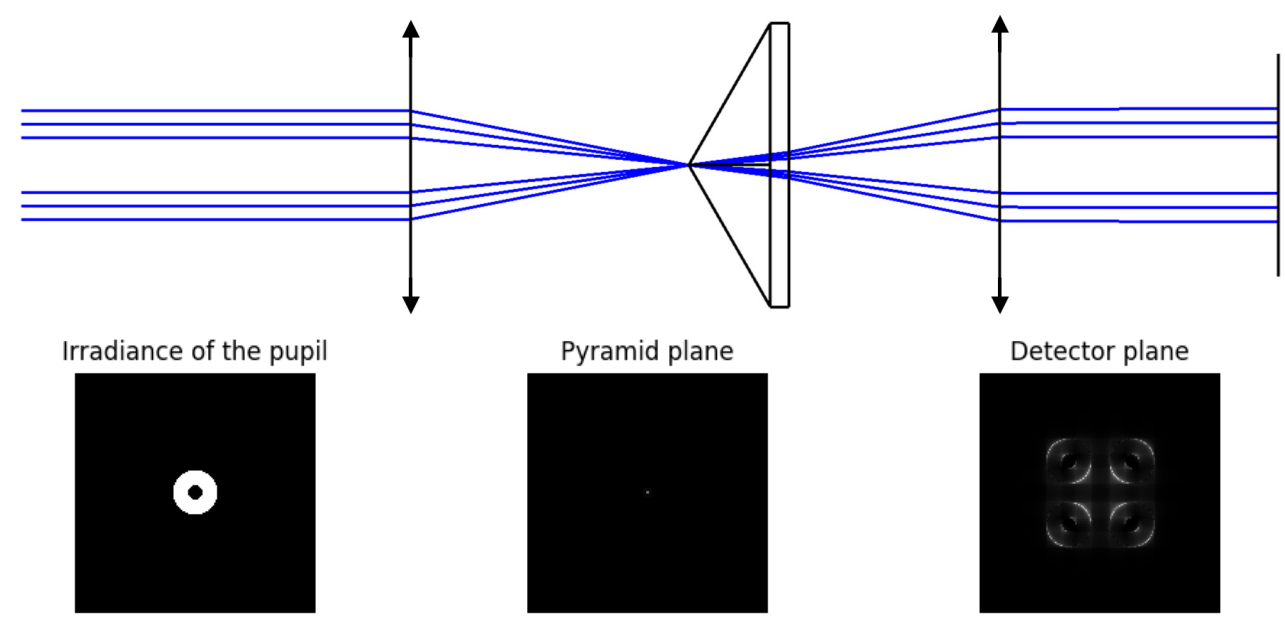

Figure 1. Ray tracing description of the pyramid wavefront sensor. Light from the telescope pupil is focused on the apex of the pyramid by a lens. Another lens is used to image the pupil onto a detector. The irradiance at each plane of interest is illustrated.

The principle behind the pyramid wavefront sensor is illustrated in Figure 1 by a simple ray tracing diagram. When considering diffraction effects as described by Fourier optics, the underlying principle of the sensor is the use of Fourier filtering to translate phase difference within the pupil to amplitude difference that can be recorded by a detector. This principle was first used and described by Foucault by using a knife edge to descriminate incoming light at the focus of a telescope mirror. The use of a prism, in this case a pyramid, allows to image the sub-pupils using this kind of discrimination without blocking any light. The distribution of the light incident on the prism can be expressed with Fourier optics by:

$$
I\left(x^{\prime}, y^{\prime}\right)=\left|E\left(x^{\prime}, y^{\prime}\right)\right|^{2}=\left|\mathcal{F} \mathcal{T}\left[p(x, y) \cdot e^{-i \phi(x, y)}\right]\right|^{2}
$$

Where $x, y$ are the pupil coordinates and $x^{\prime}, y^{\prime}$ are the image coordinates. $P(x, y)$ is the pupil function and $\phi(x, y)$ the wavefront phase. For a circular aperture of size D it is known that:

$$
I\left(x^{\prime}, y^{\prime}\right)=I_{0}\left|\frac{2 J_{1}\left(\frac{\pi r^{\prime}}{\lambda f / \#}\right)}{\left(\frac{\pi r^{\prime}}{\lambda f / \#}\right)} \circledast \mathcal{F} \mathcal{T}\left[e^{-i \phi(x, y)}\right]\right|^{2}
$$

Where $I_{0}$ is the peak intensity of the signal, $\circledast$ the convolution operator, $J_{1}$ the first order Bessel function and $r^{\prime}$ the image radial coordinates. The right hand side of the equation correspond to the airy function which discribes the intensity distribution. It can be seen that most of the light is at the apex of the pyramid (since the distribution is leaded by the airy distribution for small wavefront error). To get the distribution at the detector, we use another Fourier transform and add the phase difference induced by the pyramid:

$$
I(x, y)=I_{0}\left|\left(\frac{2 J_{1}\left(\frac{\pi r^{\prime}}{\lambda f / \#}\right)}{\left(\frac{\pi r^{\prime}}{\lambda f / \#}\right)} \circledast \mathcal{F} \mathcal{T}\left[e^{-i \phi(x, y)}\right]\right) \cdot \Pi\left(x^{\prime}, y^{\prime}\right)\right|^{2}
$$

With $\Pi\left(x^{\prime}, y^{\prime}\right)$ being the phase difference induced by the pyramid. It has been shown ${ }^{3}$ that the distribution intensity in the pupils can give a signal that is proportional to the wavefront error. However, in our analysis, 
we are using the whole detector plane as a signal to reconstruct the wavefront error. The signal we get is a somewhat more noisy, but gives an even ground when comparing with the system including an axicon.

The pyramid wavefront sensor was first described using an oscillating pyramid prism by Ragazzoni. ${ }^{4}$ Other systems use a form or another of dynamic modulation using a tip-tilt mirror to induce a dynamic modulation at the tip of the pyramid ${ }^{5}{ }^{6}$ The net effect of the modulation is to improve the linearity of the sensor, which enables the detection of larger aberrations, and also smooth out the imperfections of the prism over a modulation cycle. It is possible to add the modulation as a term in $\phi(x, y)$ using the simple analytical model provided. For the system at hand, as we want to compare with a simple system having very few moving parts, we will not consider dynamic modulation.

\subsection{Pyramid wavefront sensor with beam-shaping}
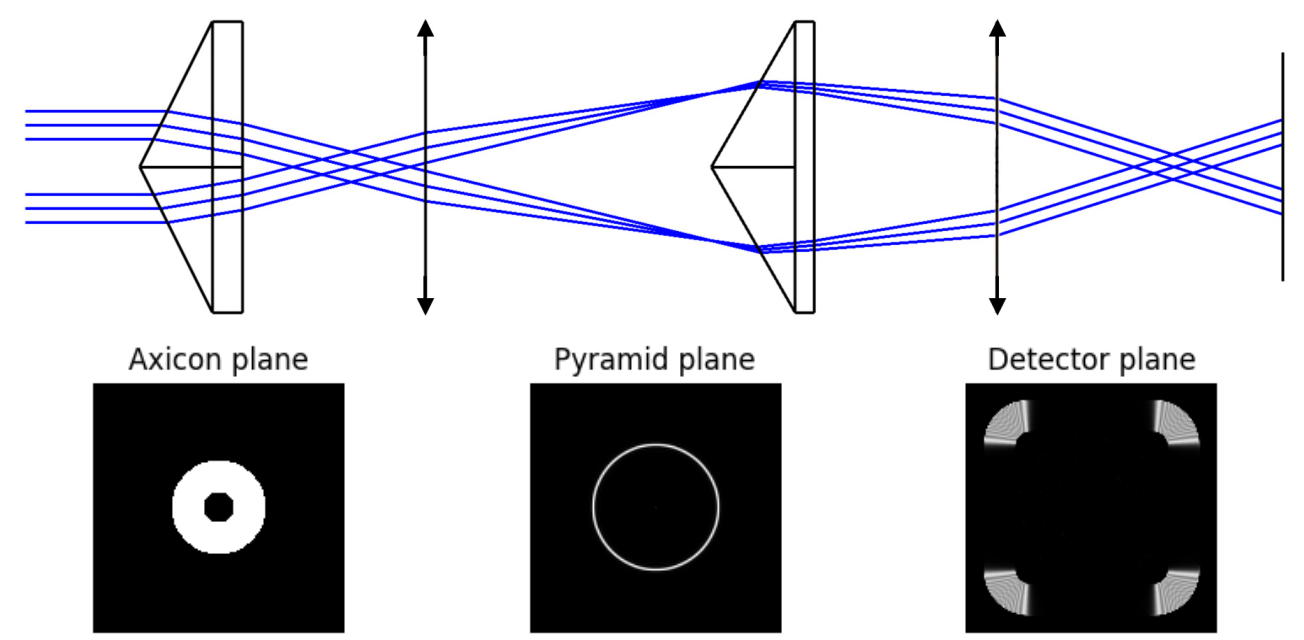

Figure 2. Ray tracing description of a pyramid wavefront sensor using an axicon as a beam shaping element. The pupil of the telescope is first imaged on the axicon and a pyramid wavefront sensor is placed after. When compared to Figure 1 it is possible to see that the light incident on the pyramid is not focused at the apex anymore.

Since manufacturing a high quality pyramid prism is difficult and the number of prisms free of defects at the apex is quite rare, it is unusual to see a system with no dynamic modulation. However, for some applications, the use of a tip-tilt mirror dedicated to the wavefront sensor can be inconvenient. In a method similar to Ragazzoni, ${ }^{7}$ which used a diffusing element placed at a conjugate pupil plane to blur the spot on the prism, we will use an optical element to modify the distribution of the light incident on the pyramid in a controlled way.

An axicon is a radially symmetrical optical element shaped into a cone (or a pyramid having an infinite number of sides). Its effect on a collimated beam is to split the PSF into a symmetrical annulus around the optical axis. It was chosen as a beam-shaping element because it is readily available as an off-the-shelf component and can be made achromatic if used in reflection. The distribution incident on the pyramid can be derived this time with:

$$
=I_{0}\left|\mathcal{F} \mathcal{T}[p(x, y)] \circledast \mathcal{F} \mathcal{T}\left[e^{-i a r}\right] \circledast \mathcal{F} \mathcal{T}\left[e^{-i \phi(x, y)}\right]\right|^{2}
$$

The second term is the phase added by the axicon (with $a$ being proportional to the angle of the axicon) and $r=\sqrt{x^{2}+y^{2}}$. The first two terms can be solved using the properties of the Hankel transform since they are radially symmetric.

$$
=I_{0}\left|\frac{2 J_{1}\left(\frac{\pi r^{\prime}}{\lambda f / \#}\right)}{\left(\frac{\pi r^{\prime}}{\lambda f / \#}\right)} \circledast \frac{i a}{\left(4 \pi r^{\prime 2}-a^{2}\right)^{3 / 2}} \circledast \mathcal{F} \mathcal{T}\left[e^{-i \phi(x, y)}\right]\right|^{2}
$$


As a first approximation, the second term can be simplified to a dirac delta because it is approximately 0 except at the discontinuity at $r^{\prime}=\sqrt{\frac{a}{4 \pi}}$ :

$$
\begin{aligned}
& =I_{0}\left|\frac{2 J_{1}\left(\frac{\pi r^{\prime}}{\lambda f / \#}\right)}{\left(\frac{\pi r^{\prime}}{\lambda f / \#}\right)} \circledast i a \delta\left(r-\sqrt{\frac{a}{4 \pi}}\right) \circledast \mathcal{F} \mathcal{T}\left[e^{-i \phi(x, y)}\right]\right|^{2} \\
& I\left(x^{\prime}, y^{\prime}\right)=I_{0}\left|\frac{2 i a J_{1}\left(\frac{\pi\left(r^{\prime}-\sqrt{\frac{a}{4 \pi}}\right)}{\lambda f / \#}\right)}{\left(\frac{\pi\left(r^{\prime}-\sqrt{\frac{a}{4 \pi}}\right)}{\lambda f / \#}\right)} \circledast \mathcal{F} \mathcal{T}\left[e^{-i \phi(x, y)}\right]\right|^{2}
\end{aligned}
$$

An illustration of this distribution is at Figure 2, at the pyramid plane. The net effect is that no light is incident on the apex of the pyramid, which makes the system to act similar to a pyramid wavefront sensor with static modulation. This way, most of the defects present on the apex of the prism can be ignored because next to no light is incident on it. Moreover, when used in a system having a central obscuration (like in Richtey-Chrétien or Schmidt-Cassegrain telescopes), no light is incident on the apex of the axicon, which also presents defects caused by manufacturing challenges. The combination of these two components minimize the light that is diffused by the defects. However, the spatial modulation changes the behavior of the wavefront sensor by shifting all light to the corners of the imaged pupil. This rises a challenge regarding the wavefront reconstruction: the signal typically used in pyramid wavefront sensor is not present. As for the pyramid wavefront sensor, we will use the entire intensity distribution on the detector to reconstruct the phase.

\section{SIMULATIONS}

\subsection{Description}

To ease the development of the beam-shaping wavefront sensor, an object-oriented python code was written as a platform to test out multiple phase shifting optical elements. The core of the code is based around Fourier optics and uses the Fourier transform as a mean of propagating light through the system. The use of python objects allows the code to function like building blocks where each optical element is it's own object and can inherit properties from other similar elements. It's a very versatile code that can be used to take into effect limited photon count, detector noise or chromatic dispersion. Take for example the system described in Section 2.2. The intensity on the detector plane is computed as:

$$
E(x, y, \lambda)=\operatorname{FFT}\left[\operatorname{FFT}\left[p(x, y, \lambda) \cdot A(x, y, \lambda) \cdot e^{-i \phi(x, y, \lambda)}\right] \cdot \Pi\left(x^{\prime}, y^{\prime}, \lambda\right)\right]
$$

Where FFT is the fast Fourier transform and $A(x, y)$ is the pupil mask (in our case an axicon) used for the simulation. The intensity at this plane is then computed as:

$$
I(x, y)=\sum_{\lambda}\left|\operatorname{FFT}\left[\operatorname{FFT}\left[p(x, y, \lambda) \cdot A(x, y, \lambda) \cdot e^{-i \phi(x, y, \lambda)}\right] \cdot \Pi\left(x^{\prime}, y^{\prime}, \lambda\right)\right]\right|^{2}
$$

The phase reconstruction algorithm is kept simple to level the two wavefront sensors on the same ground. We first record each aberration independently to construct an influence matrix which correspond to the unique intensity distribution of each Zernike polynomials. The mode is then reconstructed using:

$$
a_{i}=P_{i j} . I(x, y)_{j}
$$

Where $P_{i j}$ is the pseudo inverse of the influence matrix with $i$ modes, and for a window of $j$ pixels and $a_{i}$ is defined such as

$$
\phi_{r}(x, y)=\sum_{i} a_{i} Z_{i}
$$

With $Z_{i}$ defined as the $i^{\text {th }}$ Zernike polynomial. 


\subsection{Comparison between the two wavefront sensors}

In Section 2 we covered the analytic foundation of the pyramid wavefront sensor and a pyramid wavefront sensor using static modulation provided by an axicon. We will compare these two wavefront sensors using three criterias: reconstruction accuracy, linearity and noise propagation. Reconstruction accuracy is, for this preliminary analysis, only a qualitative measure. It only checks if the sensor is able to reconstruct complex aberrations. As for linearity, this criteria verifies the claim that if the wavefront aberration is doubled, that the signal (and the reconstruction) will be doubled too. And finally, the noise propagation correspond to the variance of the residual aberration caused by the reconstruction. This last parameter gives us an idea of the sensitivity of the system (the lower the variance, the better).
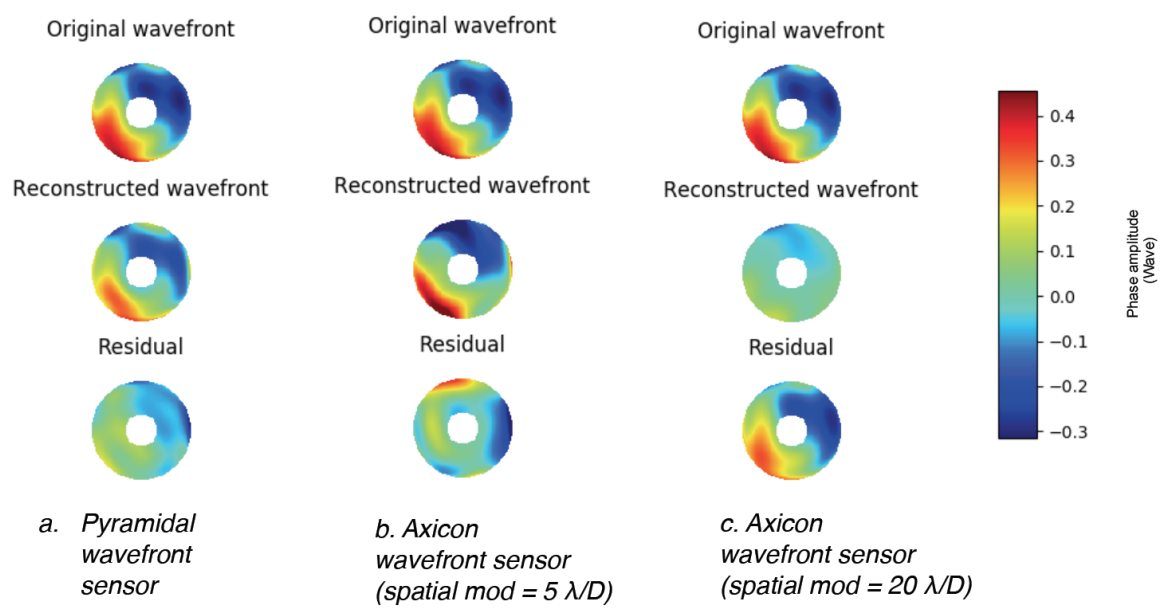

Figure 3. Reconstruction and residual on three different configuration of spatial modulation. The spatial modulation is provided by an axicon (and is computed as the annulus radius incident on the pyramid). The results from the reconstruction correspond to one iteration of reconstruction.

Without any surprises, if the aberrations are kept within the linearity range, the pyramid wavefront sensor is able to accurately reconstruct the wavefront errors present in the pupil as illustrated in Figure 3. It is also possible to see that when the modulation is kept small, the aberrations that are being correctly detected by the non-modulated pyramid wavefront sensor are mostly reconstructed. However, when the modulation is too important, the faint aberrations are not detected at all by the wavefront sensor since the sensibility has been shifted to higher amplitude aberrations.
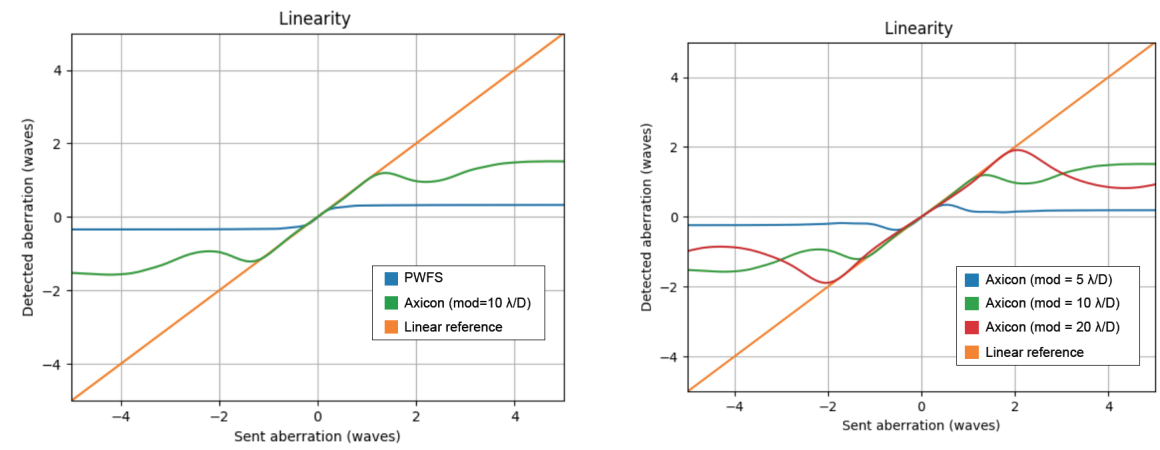

Figure 4. Linearity plot corresponding to the signal read by the different wavefront sensors for a given amplitude in the pupil. These were done using Tilt $\left(Z_{2}\right)$ 
Figure 4 shows how the linearity is increased with static modulation provided by an axicon. The linearity of the pyramid wavefront sensor is quite limited to very faint aberrations and adding the axicon can extend this linearity to what is required by the application. The downside is that the behavior outside the linearity range is less predictable than what is seen with dynamic modulation or no modulation at all.

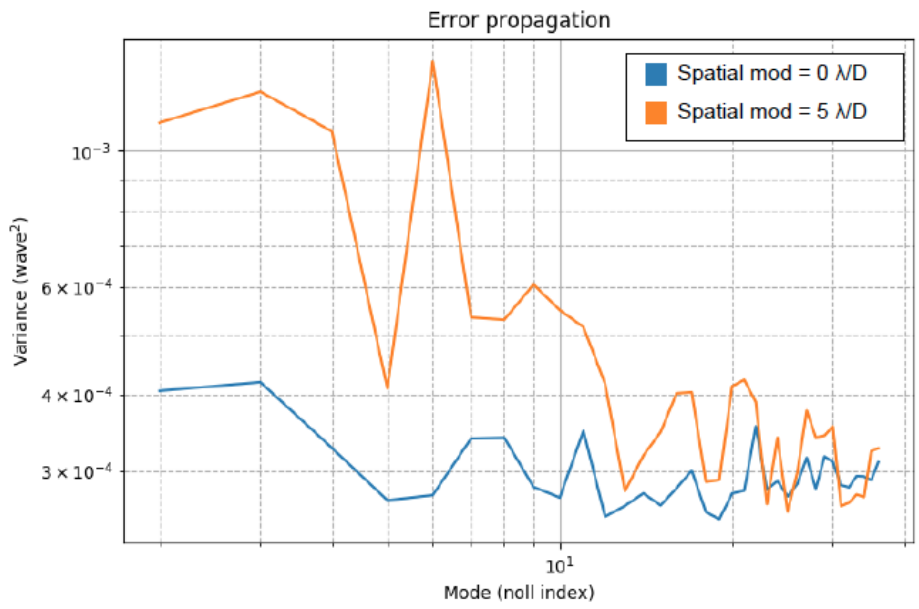

Figure 5. Noise propagation for the two wavefront sensors at hand.

The modulation induced by the axicon increases the noise present when reconstructing lower order modes. The behavior, illustrated in Figure 5 represents, as explained earlier, the variance of the residual for a flat wavefront. This modal distribution of this noise is similar to what we would see for a dynamically modulated wavefront sensor, with a higher component within the lower Zernike modes.

Overall, it is possible to see that the spatial and static modulation induced by the axicon doesn't break the behavior of the pyramid wavefront sensor, and is similar to what it would be possible to see with dynamic modulation. However we did not characterize the reconstruction efficiency and did not compare to a pyramid wavefront sensor using a reconstruction scheme similar to what is present in the literature.

\section{LABORATORY TESTING}

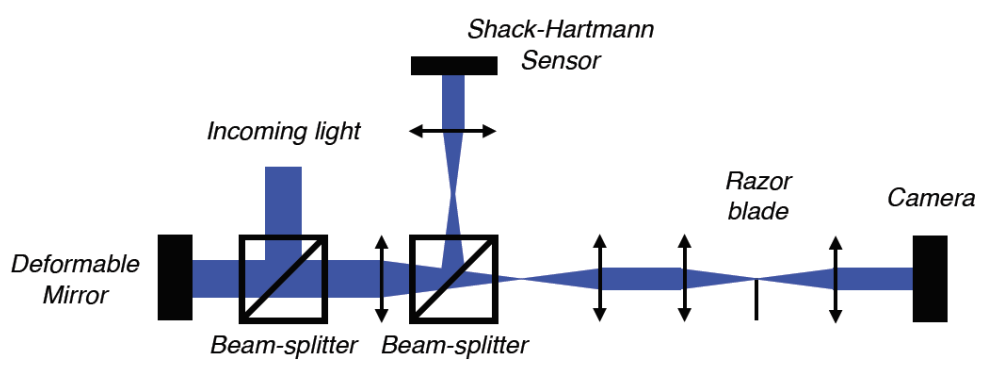

Figure 6. Illustration of the optic path for the setup used. The deformable mirror and Shack-Hartmann can work in closed-loop, and the foucault knife is placed in the science path of the setup.

A prototype of the beam-shaping pyramid wavefront sensor was designed by modifying an existing adaptive optics test bench. Because no pyramid was available at the time, a moving razor blade was used as a way to split the image plane beam. As it is possible to see from Section 2.1, this is similar to what is done using a foucoult test using a knife edge. This gives us access to a one dimensional version of the pyramid wavefront sensor as long as we rotate the knife edge to get access to the light that was blocked in an earlier configuration. This setup makes 
it possible to reconstruct aberrations in the same way that was described in Section 3. Different aberrations can be generated by closing the adaptive optics loop using the ALPAO DM-97 and the Shack-Hartmann sensor. The axicon phase shift is added numerically to the aberration induced by the deformable mirror.

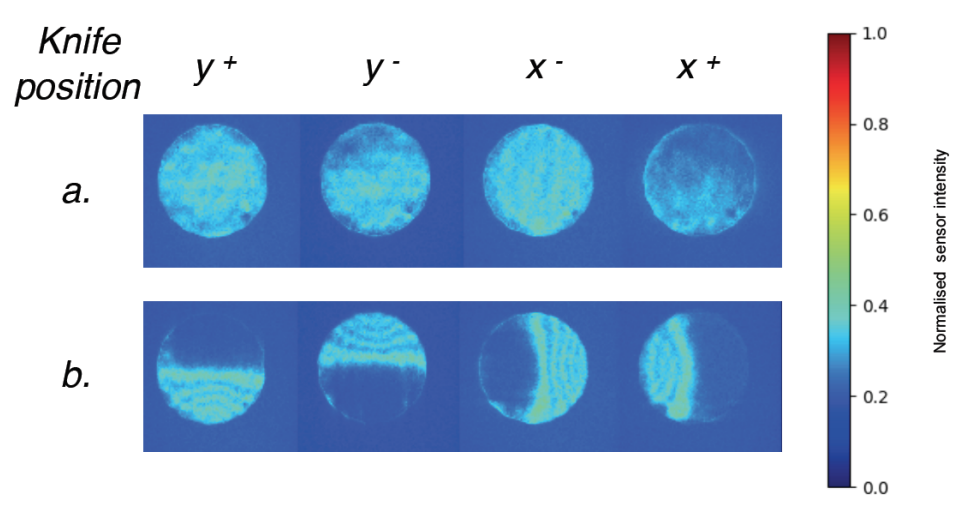

\section{a. Conventional pyramid $\quad$ b. Added axicon in pupil}

Figure 7. Intensity recorded on the camera with and without axicon for different knife position.

Minimal analysis was done since the setup was running slow and imprecise due to the knife rotation. As we can see in Figure 7, the behavior of the system if very similar to what was predicted by the simulation in Figure 2. We can see that most of the light is shifted to the extremities of the pupil, which means that the signal processing similar to a conventional pyramid wavefront sensor cannot be used. Preliminary results show that reconstruction is possible, and that the linearity of the signal is increased as predicted by simulations. A more in depth analysis will need to be performed to get repeatable results from a prototype.

\section{NEXT STEPS}

\subsection{On-sky prototype at OMM}

An prototype version of the wavefront sensor is currently being designed and will be tested at the $1.6 \mathrm{~m}$ telescope of the Observatoire du Mont-Mgantic using Universit Lavals AO test bench. Universit Lavals on-sky AO test-bench ${ }^{8}$ is designed to directly compare two types of wavefront sensors on-sky at the same time. A Shack-Hartmann sensor will be used for closing the loop on the sky while leaving controlled residual levels of low-order aberrations to be measured by the beam-shaping pyramid sensor. This will provide valuable data as to the performance of this kind of wavefront sensor.

\section{$5.2 \mathrm{HiCIBaS}$}

The High Contrast Imaging Balloon System $(\mathrm{HiCIBaS})$ is a balloon-borne telescope developed by the our group at Université Laval. The mission is focused on testing new optical components for future high contrast missions in space-like conditions. The first goal is to record the behavior of atmospheric turbulence at a $35 \mathrm{~km}$ altitude using a wavefront sensor. The combination of an axicon and a pyramid wavefront sensor makes it interesting for this type of mission because it is free of moving components, retains the attributes of a pyramid wavefront sensor and increases linearity response. The mission will also house a stellar coronagraph that will also test a deformable mirror in open loop with the LOWFS.

\section{CONCLUSION}

We investigated the use of an axicon in a pyramid wavefront sensor as a mean to replace dynamic modulation for small amplitude and low order wavefront aberrations. It was possible to see that even with the introduction of a known dominant non-common path aberration it is still possible to reconstruct wavefront error accurately. 
Moreover, we can control the linearity range of the wavefront sensor by changing the angle of the axicon. This allows it to be finely tuned to the requirement of a high-contrast imaging system. Also, by replacing all refractive optical elements by reflective elements, it could be possible to make this system completely achromatic.

There is still much testing and analysis to be done, but these early results show a promising approach. Further analysis will be done in the following months before the final integration in the HiCIBaS project.

\section{AKNOWLEDGEMENT}

- The HiCIBaS project is supported by the FAST grant from CSA that funds university research project on spacelike missions.

- Nuvu cameras EMCCD are used as the main camera for the LOWFS that will be used on the HiCIBaS project.

\section{REFERENCES}

[1] Dimitri Mawet, Laurent Pueyo, P. L. L. M. W. T. A. B. J. T. T. S. G. E. S. J. M. R. B. M. K. P. B. B. M. C. M. B. O. H. B. J.-L. B. N. D. J. G. O. G. J. K. B. M. D. M. N. M. L. P. D. S. C. V. J. K. W., "Review of small-angle coronagraphic techniques in the wake of ground-based second-generation adaptive optics systems," (2012).

[2] Guyon, O., "Imaging earth-like planets around late-type stars with low-inner working angle piaa coronagraphy," (2013).

[3] Riccardi, A., Bindi, N., Ragazzoni, R., Esposito, S., and Stefanini, P., "Laboratory characterization of a foucault-like wavefront sensor for adaptive optics," in [Adaptive Optical System Technologies], Bonaccini, D. and Tyson, R. K., eds., SPIE (sep 1998).

[4] Ragazzoni, R., "Pupil plane wavefront sensing with an oscillating prism," Journal of Modern Optics 43, 289-293 (feb 1996).

[5] Martin, O., Véran, J.-P., Anctil, G., Bourqui, P., Châteauneuf, F., Gauvin, J., Goyette, P., Lagacé, F., Turbide, S., and Wang, M., "Pyramidal wavefront sensor demonstrator at INO," in [Adaptive Optics Systems IV], Marchetti, E., Close, L. M., and Véran, J.-P., eds., SPIE (aug 2014).

[6] Hadi, K. E., Vignaux, M., and Fusco, T., "Development of a pyramid wave-front sensor," Development of a Pyramid Wave-front Sensor (Dec 2013).

[7] Ragazzoni, R., Diolaiti, E., and Vernet, E., "A pyramid wavefront sensor with no dynamic modulation," Optics Communications 208, 51-60 (jul 2002).

[8] Brousseau, D., Thibault, S., Lavigne, J.-F., and Véran, J.-P., "On-sky AO test bench," in [Adaptive Optics Systems V], Marchetti, E., Close, L. M., and Véran, J.-P., eds., SPIE (aug 2016). 